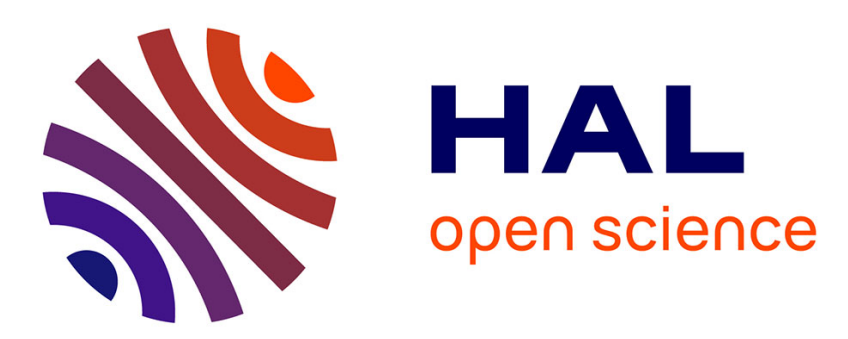

\title{
Improvement of DDM pre-processing for the simulation of the GRAVES radar densified sparse array for Space Surveillance and Tracking
}

André Barka, Antoine Jouadé

\section{To cite this version:}

André Barka, Antoine Jouadé. Improvement of DDM pre-processing for the simulation of the GRAVES radar densified sparse array for Space Surveillance and Tracking. IEEE Antennas and Wireless Propagation Letters, In press, 10.1109/LAWP.2020.3004925 . hal-02907224

\section{HAL Id: hal-02907224 \\ https://hal.science/hal-02907224}

Submitted on 27 Jul 2020

HAL is a multi-disciplinary open access archive for the deposit and dissemination of scientific research documents, whether they are published or not. The documents may come from teaching and research institutions in France or abroad, or from public or private research centers.
L'archive ouverte pluridisciplinaire HAL, est destinée au dépôt et à la diffusion de documents scientifiques de niveau recherche, publiés ou non, émanant des établissements d'enseignement et de recherche français ou étrangers, des laboratoires publics ou privés. 


\title{
Improvement of DDM pre-processing for the simulation of the GRAVES radar densified sparse array for Space Surveillance and Tracking
}

\author{
A. Barka, A. Jouadé
}

\begin{abstract}
In a recent work [1] we have discussed our implementation of the Finite Element Tearing and Interconnecting method (FETI-2LM) a Domain Decompositon Method (DDM) for the full-wave simulation of the large GRAVES sparse array (60 $\mathrm{m}$ diameter disk). Due to the non-regular distribution of the antennas of the sparse array, such simulations in reasonable times are not accessible with existing FETI methods optimized for repetitive geometries and who do not benefit from an effective parallelization technique. A particularity of the pre-processing step of our DDM is that the complete sparse array mesh is not built upstream of the resolution of the electromagnetic problem. Each sub-domain in our decomposition is equipped with its own local mesh, belonging to a limited set of unit-cell meshes, generated separately. Initially, a total of 13,692 sub-domains were used chosen among 3 types of sub-domains, considering those corresponding to antennas (200), air sub-domains (800) and ground plane sub-domains $(12,592)$. This simulation has required 13, 692 Intel Xeon Broadwell E5-2680v4 processing cores equiped with 4 GB of memory, each handling one sub-domain. A new strategy is shown that requires only 2408 cores for the same simulation, leading to a $82.41 \%$ reduction of the required cores, as well as a $30 \%$ reduction of the simulation time.
\end{abstract}

Index Terms-Aerospace control, Bi-static Radar, Doppler radar, Low earth orbit satellites, Parallel machines, Supercomputers, Radar Tracking, Space Surveillance and Tracking, STT, Sparse array, domain decomposition, Finite Element Tearing and Interconnecting (FETI)

\section{INTRODUCTION}

$\mathbf{S}$ PACE Surveillance and Tracking (SST) systems permit to build a space picture while maintaining a catalog of Low Earth Orbit (LEO) objects. Graves System (Grand Réseau Adapté pour la VEille Spatiale = Large Radar Array for Space Survey) [2], owned by the French Ministry for the Armed Forces, is a bistatic continuous-wave (CW) radar with a coverage forming a fence in the sky. It ensures the detection of each LEO object, at least once every 24 hours.

The study deals with the densification of the receiving array (Fig. 1) by investigating, through full-wave electromagnetic simulation, the coupling effects of a more populated sparse array on the overall array performance.

Originally developed for acoustics and helmholtz equation [3], FETI methods have been successfully extended to the

This work was supported in part by the French Armament General Directorate (Direction Générale de l'Armement) through the contract for the GRAVES refurbishment and upgrades with a partial EU SST Program, and in part by the Grand Equipement National de Calcul Intensif (GENCI) Grant c2014109083.

The Authors are with ONERA/DEMR, Université de Toulouse, France ONERA is the French Aerospace Lab and DEMR its ElectroMagnetism and Radar Department. (e-mail: andre.barka@onera.fr, antoine.jouade@onera.fr).

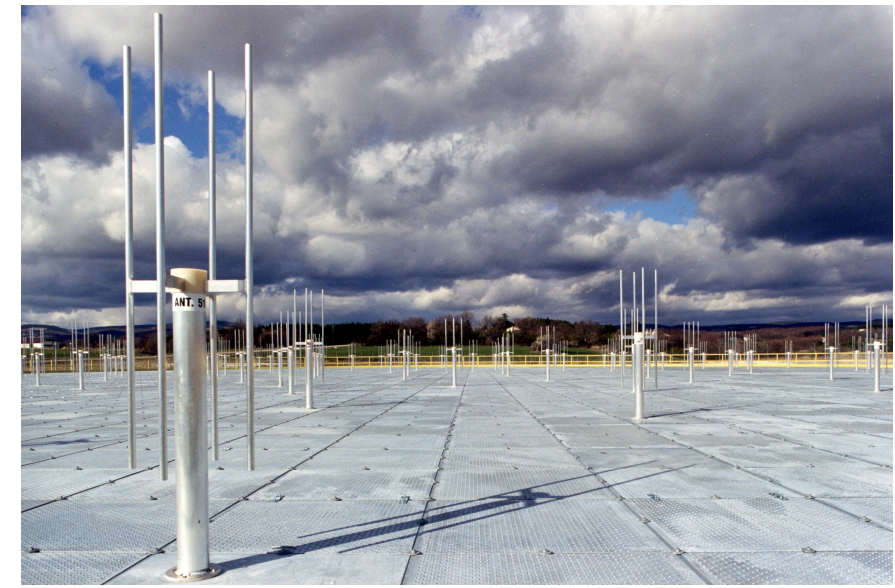

Fig. 1: GRAVES sparse receiving array [1]

solution of Maxwell equations (FETI-2LM) in the frequency domain for the calculation of antenna arrays and metamaterial periodic structures [4], [5], [6]. These previous works are optimized for problems with geometric repetitions and discussed for single antenna point source [5] or aperture source where all the radiating elements are excited to ensure broadside or non-broadside scan [6]. These methods cannot be used for the simulation of non-regular grids encountered in the GRAVES sparse arrays. Indeed, the GRAVES array has been determined by applying a process of approximating a desired continuous current distribution by populating the aperture with $\mathrm{N}$ discrete antenna elements and an optimization procedure is then be performed to prevent shadowing. The initial array configuration is thus a non-repetitive structuresunflower array [7], and then, the radii (from the center of the aperture) are modified using the optimization procedure (maintaining the side lobe level almost unchanged while modifying the antenna angular coordinate). In addition, the efficiency of [4], [5], [6] on parallel machines using several thousand computing cores (mandatory for simulation of the $60 \mathrm{~m}$ diameter disk of the GRAVES sparse array) has not been demonstrated. Remenber we have to calculate the surrounded radiation patterns of the 200 antennas populating the array as well as the array scattering matrix. As the same problem must be solved with the 200 antennas, one drawback of the iterative methods implemented in [4], [5], [6] is that they generally need to restart from scratch for each new antenna as they are not equipped with Krylov recycling techniques. Complementary 
developments for effectively treating multiple sources with existing recycling Krylov techniques have been proposed in [8] but the effectiveness has only been demonstrated for incident plane waves (linearly dependent right hand-side). This very favorable situation is not encountered for the GRAVES array simulation for which the port excitation by TEM modes is generating higly different solutions not able to fill in an efficient recycling Krylov basis .

Recently [1], to overcome the calculation difficulties indicated above, the simulation of the large size GRAVES sparse array has been performed with the Finite Element Tearing and Interconnecting using two Lagrange multipliers (FETI-2LM) technique [3], [9], [10] implemented in ONERA's FACTOPO code. The originality is that the complete mesh is not built upstream of the resolution of the electromagnetic problem avoiding the use of specific mesh splitter tools such as METIS [11] to decompose the large scale initial mesh. The method is general, based on parallel-thinking, and tailored to efficiently introduce 3D non-periodic grids with sub-domains that are not necessarily of the same size and not necessarily populating the array with repetitive blocks. This method has also demonstrated its effectiveness for simulating linearly independent multi-source (TEM mode feeds) problems with the implementation of Block Krylov Recycling Strategy (BKRS) and improves significantly the previous works of [4], [5] and [6]. The efficiency of the method for linearly independent feeds (in our application the 200 surrounded antenna near fields are significantly different) as already been demonstrated in [9] for array antennas where gains in computation time of around 11 have been observed. Each sub-domain of our decomposition belonging to a limited set of unit-cell meshes (antenna, air and groud plane subdomains), is locally meshed with an automatic procedure of the GID preprocessor [12] based on GID batch files and $T c l$ scripts [13]. This way, very complex antennas are efficiently represented with only a few local meshes. The interest of this strategy is that since the method is implemented on parallel machines, each computing core is allocated exclusively to a sub-domain. Then, with at least 4 GB of memory allocated exclusively to a sub-domain, relatively fine meshes can be implemented easily in each subdomain. This methodology is then rendering unnecessary the sometimes very slow adaptive mesh refinement techniques. The paper is organized as follows. Section II presents the pre-processing strategy implemented to decompose the 200 antennas GRAVES sparse array with a limited set of local meshes and the improvement proposed to considerably reduce the number of computational cores necessary to solve the problem. Finally, section III discusses the simulation performances of the new FETI-2LM implementation applied to the GRAVES sparse array.

\section{THE SUB-DOMAIN DECOMPOSITION}

One main particularity in our implementation remains in the fact that we do not mesh the initial global sparse array before the resolution of the electromagnetic problem. The global array is geometrically broken down in few generic sub-domains (3 unit-cell in [1]) each equipped with its own local mesh.

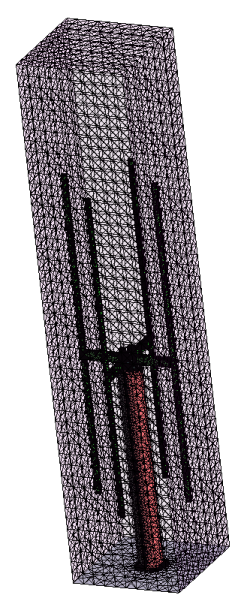

Fig. 2: "Antenna" sub-domain $(730,179$ tetrahedra, 765,425 edges)

They are generated separately with an automatic procedure of the GID preprocessor and as a consequence we do not use METIS [11] for partitionning the large scale initial mesh into sub-domains. Thus the geometry of very complex sparse array antennas is represented efficiently with only a few local meshes. Since the method is implemented on parallel machines, each computing core with at least 4 GB of memory is allocated exclusively to a sub-domain (elementary cell of the sparse array). The strategy used in a recent work [1] was to populate a regular grid of regular periodicity with three types of sub-domains:

1) Antenna sub-domain: It embeds the antenna geometry with surrounding Absorbing Boundary Conditions (ABC) and a Perfect Electrical Conductor (PEC) at $\mathrm{z}=0$ as shown in Fig. 2.

2) Ground sub-domain: It is an empty sub-domain with the same boundary conditions as the antenna sub-domain.

3) Air sub-domain: It is an empty sub-domain with $\mathrm{ABC}$ boundary conditions.

This allowed to virtually build the mesh of the complete sparse array with only 3 sub-domain meshes. These generic sub-domains were then duplicated and translated to generate the complete sparse array. It was decided to use sub-domains of size $\mathrm{x}=50 \mathrm{~cm}, \mathrm{y}=50 \mathrm{~cm}$ and $\mathrm{z}=2.5 \mathrm{~m}$. The size of 50 $\mathrm{cm}$ corresponds to a limit related to the size of the antenna element. Such strategy has permitted to have 200 "antenna" subdomains, 12,592 "ground" sub-domains and 800 "air" sub-domains. This simulation required 13,692 Intel Xeon Broadwell E5-2680v4 processing cores, each handling one sub-domain. The subdomain decomposition over a regular grid is shown in Fig. 4. (left side).

A new strategy is proposed in this work to drastically reduce the number of core required for the simulation of the overall sparse array with an equivalent accuracy. As each sub-domain is handled by a single core of a limited 4-GB memory, it is obvious that the "antenna" sub-domain is discretized with much more tetrahedra elements than the "Ground" or "Air" sub-domains. The new strategy consists in grouping together several "ground" and "air" sub-domains and assigning them 


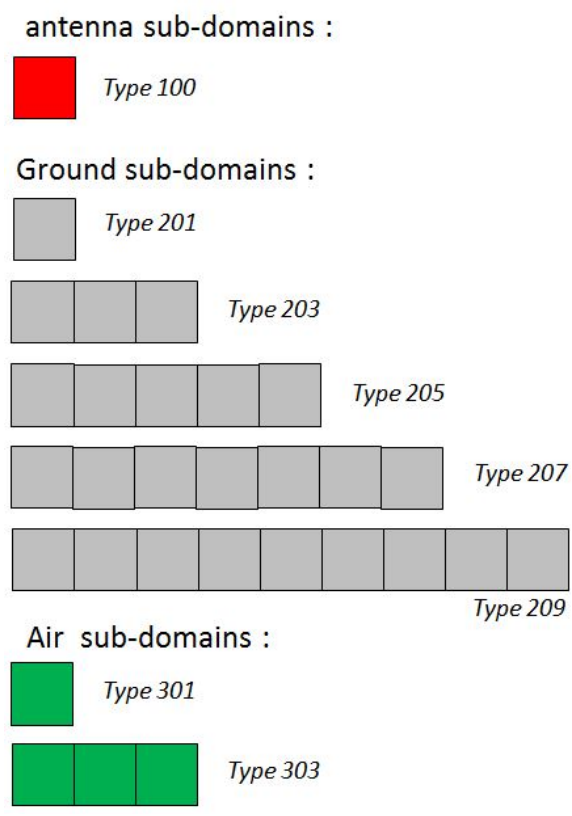

Fig. 3: Schematic of the 8 sub-domains

to a single core by keeping in mind the limited memory. Instead of having only 3 different sub-domains of same size, 8 different sub-domains of different sizes have been specified as shown in Fig. 3. The biggest sub-domain is composed by 9 initial "ground" sub-domains of size $50 \mathrm{~cm} \mathrm{x} 50 \mathrm{~cm}$ x $2.5 \mathrm{~m}$. The linear arrangement has been chosen because it is better suited to drastically reduce the number of required cores to simulate the overall sparse array. Hence, the new strategy consists in populating the regular grid of regular periodicity with eight types of sub-domains with different sizes. To build the entire grid with the eight sub-domains while minimizing the number of required cores, an algorithm is used filling in the biggest sub-domains first over the entire grid before filling in a smaller one. The result is shown in Fig. 4 (right side). The new strategy allows a significant reduction in the number of cores necessary for the entire simulation from 13,692 to only 2,408 , leading to a $82.41 \%$ reduction of the required cores. The entire grid is filled as follows (Tab. I): 200 "antenna" sub-domains, 1258 "macro ground made of 9 initial cells" sub-domains, 80 "macro ground made of 7 initial cells" sub-domains, 75 "macro ground made of 5 initial cells" sub-domains, 72 "macro ground made of 3 initial cells" sub-domains, 219 "macro ground made of 1 initial cell" subdomains, 148 "macro air made of 3 initial cells" sub-domains and 356 "macro air made of 1 initial cell" sub-domains.

\section{PARALLEL EFFICIENCY}

The computation statistics are given in Tab. II and a comparison between the previous strategy and the new one is performed. Optimizing the size of the sub-domains has made it possible to considerably reduce the total number of unknowns as well as the size of the interface problem. The total number of unknowns in the 3D sparse array computational domain is 0.65 billions and the number of unknowns on the skeleton interface is reduced to 91.45 millions. Due to the smaller size of the skeleton interface, the problem was solved through 160 iterations in 12 minutes per antenna with a stop criteria of $10^{-2}$ corresponding to a $30 \%$ reduction of the simulation time (17 minutes per antenna with the previous strategy). Finally, the simulation of the 200 antennas of the GRAVES sparse array with this new strategy requires a total of 133 hours on 2408 cores, corresponding to 320,264 cumulated hours. Fig. 5 shows a comparison between the radiation pattern of one of the antenna in the center of the sparse array (identified by a blue square in Fig. 4) using the previous strategy and the new one. Slight differences are expected since the mesh of the "Ground" and "Air" sub-domains is coarser in the new strategy as compared to the previous one to be handled by a unique core. It has been observed over the $3 \mathrm{D}$ radiation patterns (considering the two linear polarisations), an averaged difference of $0.13 \mathrm{~dB}$ with a maximum averaged difference of $0.21 \mathrm{~dB}$. However, this is a tolerable difference in proportion to the $82.41 \%$ reduction of the required cores permitted by the new strategy to simulate the overall sparse array.

TABLE I: Sub-domains unknowns

\begin{tabular}{|l|r|r|r|}
\hline Type & Sub-domains & local unknowns & total unknowns / type \\
\hline 100 & 200 & 765,425 & 153 millions \\
\hline 209 & 1258 & 331,488 & 417 millions \\
\hline 207 & 80 & 258,580 & 21 millions \\
\hline 205 & 75 & 185,872 & 14 millions \\
\hline 203 & 72 & 112,482 & 8 millions \\
\hline 201 & 219 & 40,261 & 9 millions \\
\hline 303 & 148 & 112,484 & 17 millions \\
\hline 301 & 356 & 40,261 & 14 millions \\
\hline TOTAL & $\mathbf{2 4 0 8}$ & & $\mathbf{0 . 6 5}$ billion \\
\hline
\end{tabular}

TABLE II: Computational statistics

\begin{tabular}{|l|r|r|}
\hline Mesh parameters (unknowns) & Previous strategy & New strategy \\
\hline Antenna sub-domain & 765,425 & 765,425 \\
\hline Skeleton interface problem & 157.6 millions & 91.45 millions \\
\hline Total unknowns of the array & 10.47 billions & 0.65 billions \\
\hline Simulation parameters & & \\
\hline Number of cores & 13692 & 2408 \\
\hline Number of iterations & 300 & 160 \\
\hline Stopping criterion & $\epsilon=10^{-2}$ & $\epsilon=10^{-2}$ \\
\hline Elapse time (per antenna) & 17 minutes & 12 minutes \\
\hline Memory peak (per core) & $3.5 \mathrm{~Gb}$ & $3.5 \mathrm{~Gb}$ \\
\hline
\end{tabular}

\section{CONCLUSION}

The new strategy applied in this paper allows a $82.41 \%$ reduction of the required cores and a reduction of $30 \%$ of the simulation time while maintaining results with good accuracy. This new strategy will benefit future developments, to accurately allocate an optimized sub-domain to a specific core with limited memory. This ensures an efficient exploitation of parallel computing architectures. This will permit to simulate bigger structures while maintaining unchanged the parallel supercomputer capability.

\section{REFERENCES}

[1] A. Jouadé and A. Barka, "Massively parallel implementation of feti-21m methods for the simulation of the sparse receiving array evolution of the graves radar system for space surveillance and tracking," IEEE Access, vol. 7, pp. 128968-128979, 2019. 


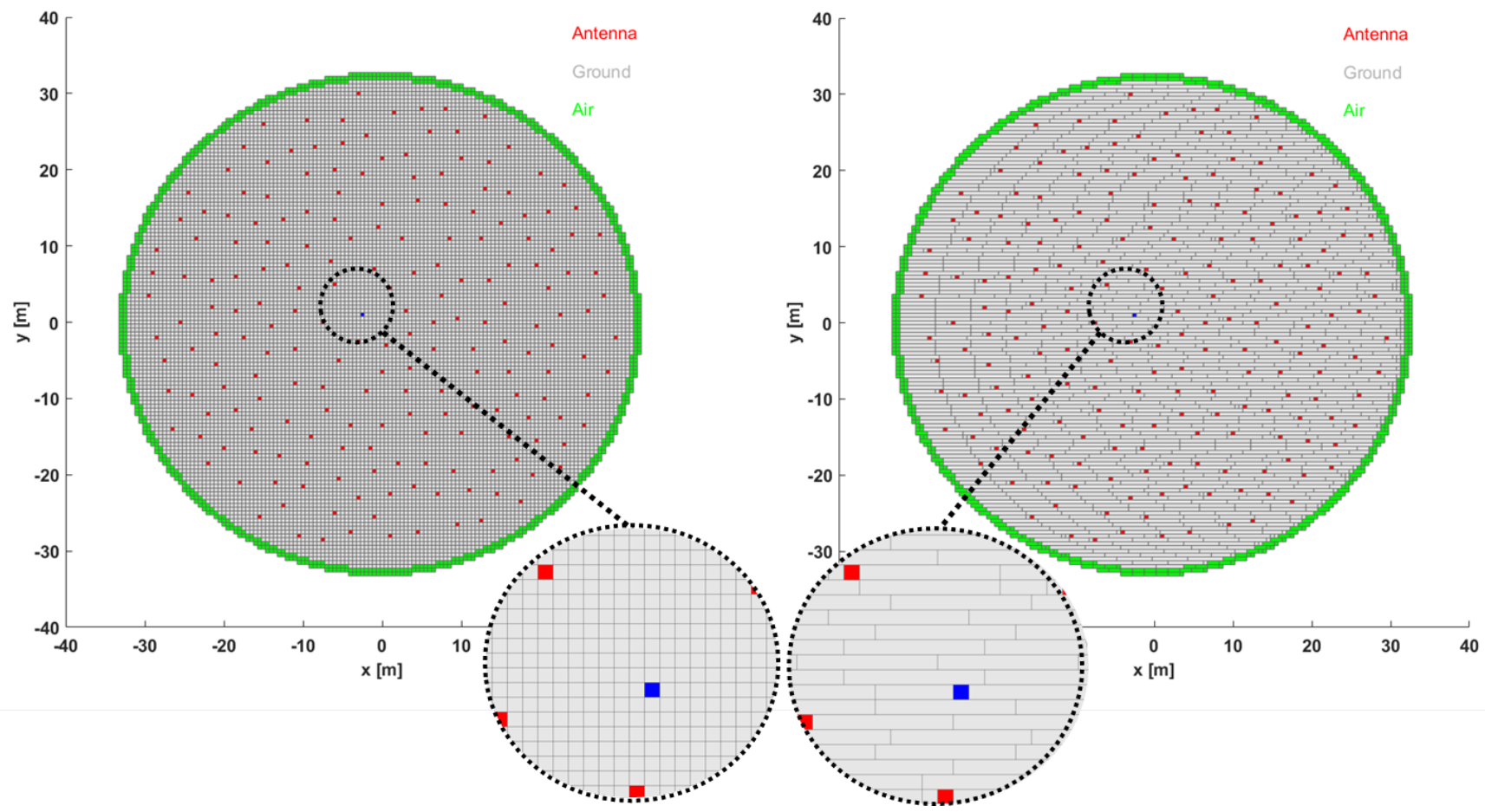

Fig. 4: Sparse array sub-domain decomposition to populate a regular grid of regular periodicity with three types of sub-domains (left side) and to populate a regular grid with regular periodicity with eight types of sub-domains (right side)
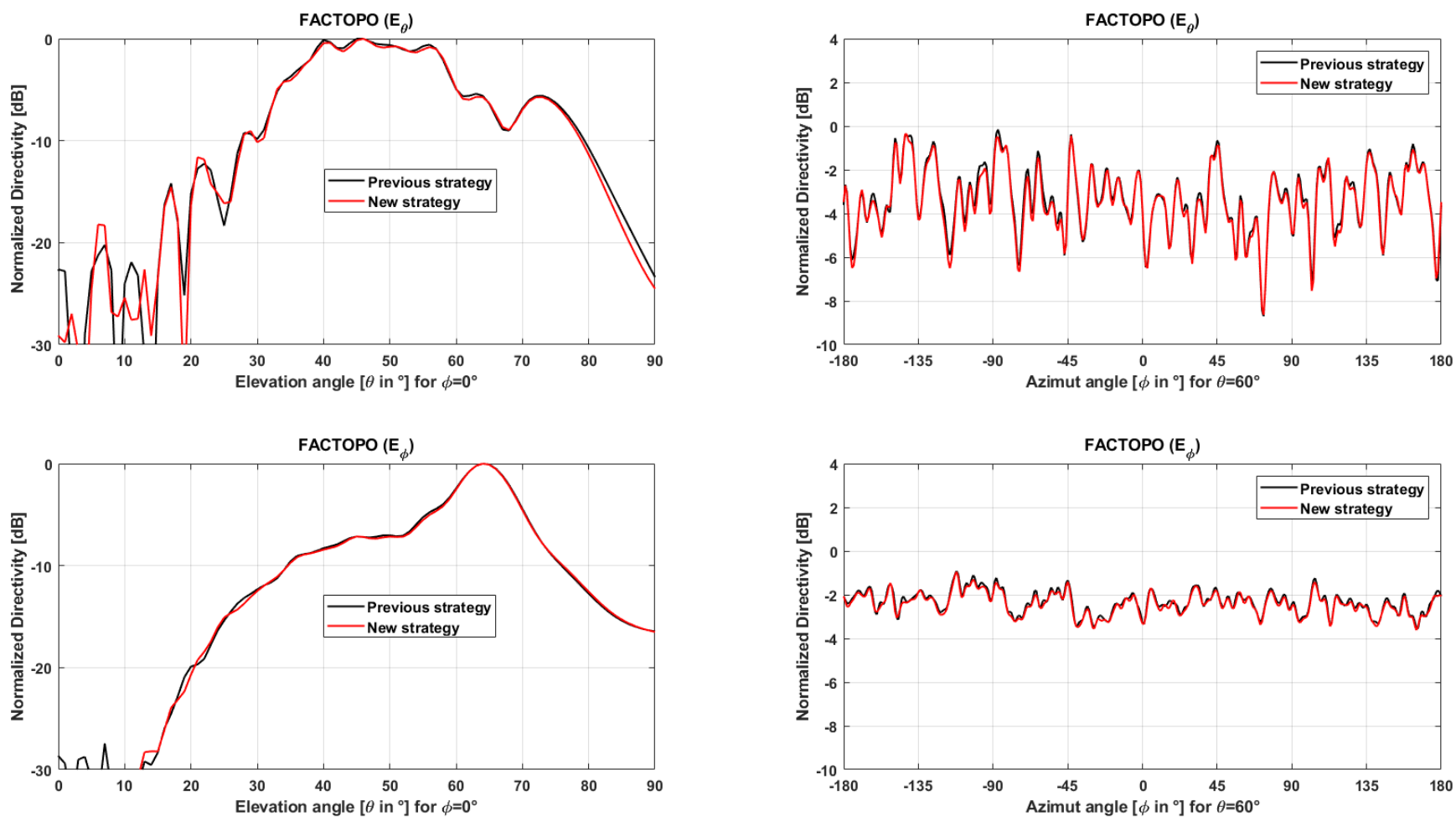

Fig. 5: Normalized radiation pattern comparison between the previous strategy and the new strategy for the two linear polarisations $\left(E_{\theta}\right.$ and $\left.E_{\phi}\right)$ in two cutting planes (Elevation plane for $\phi=0^{\circ}$ and Azimut plane for $\theta=60^{\circ}$ ).

[2] T. Michal, J. Eglizeaud, and J. Bouchard, "Graves: the new french system for space surveillance," in 4th European Conference on Space Debris, vol. 587, p. 61, 2005
[3] C. Farhat and F. Roux, "A method of finite element tearing and interconnecting and its parallel solution algorithm," Internat. J. Numer. Maths Engrg, vol. 32, pp. 1205-1227, 1991. 
[4] M. Vouvakis, Z. Zendes, and J. Lee, "A fem domain decomposition method for photonic and electromagnetic band gap structures," IEEE Trans. Antennas and Propagation, vol. 54, pp. 3000-3009, Feb 2006.

[5] K. Zhao, V. Rawat, S. Lee, and J. Lee, "A domain decomposition method with nonconformal meshes for finite periodic and semi-periodic structures," IEEE Trans. Antennas and Propagation, vol. 55, pp. 2559 2570, Sept 2007.

[6] Y. Li and J.-M. Jin, "A vector dual-primal finite element tearing and interconnecting method for solving 3-d large scale elemectromagnetic problems," IEEE Trans. Antennas and Propagation, vol. 54, pp. 721733, Oct 2006.

[7] A. Ramalli and P. Tortoli, "256-element density-tapered spiral matrices for ultrasound phased imaging," in Ultrasonics Symposium (IUS), p. 2087?2090, IEEE International, 2014.

[8] Z. Peng, M. B. Stephanson, and J. F. Lee, "Fast computation of angular responses of large-scale three-dimensional electromagnetic wave scattering," IEEE Trans. Antennas and Propagation, vol. 58, pp. 3004 3012, Sept 2010.

[9] F.-X. Roux and A. Barka, "Block krylov recycling algorithms for feti$2 \mathrm{~lm}$ applied to 3-d electromagnetic wave scattering and radiation," IEEE Transactions on Antennas and Propagation, vol. 65, no. 4, pp. 1886 1895, 2017.

[10] F. Roux and A. Barka, FETI methods Chapter, Computational Electromagnetism - Recent advances and Engineering Applications. Springer, R. Mittra, 2014

[11] "Metis - serial graph partitioning and fillreducing matrix ordering," [online] Available. http://glaros.dtc.umn.edu/gkhome/views/metis.

[12] "Gid the personel pre ans post processor," [online] Available. https://www.gidhome. com.

[13] "Tcl reference manual," [online] Available. https://www.http://tmml.sourceforge.net/doc/tcl/. 